\title{
Role of Acid Suppression in Acid-related Diseases: Proton Pump Inhibitor and Potassium- competitive Acid Blocker
}

\author{
Hideki Mori ${ }^{1}$ and Hidekazu Suzuki ${ }^{2 *}$ \\ ${ }^{1}$ Department of Gastroenterology, National Hospital Organization Tokyo Medical Center, Tokyo, Japan; and ${ }^{2}$ Fellowship Training Center and \\ Medical Education Center, Keio University School of Medicine, Tokyo, Japan
}

Proton pump inhibitors are commonly utilized for the treatment of gastric acid-related diseases, such as gastroesophageal reflux disease, peptic ulcer disease, and Helicobacter pylori infection, and for the prevention of low-dose aspirin or nonsteroidal antiinflammatory drug-induced peptic ulcers. Vonoprazan is a first-in-class potassium-competitive acid blocker, which has distinct advantages compared to other conventional proton pump inhibitors in terms of the efficacy for acid suppression. Due to its strong gastric acid suppression capabilities, vonoprazan serves as an effective drug for the treatment of gastroesophageal reflux disease and H. pylori infection.

(J Neurogastroenterol Motil 2019;25:6-14)

\section{Key Words}

Gastroesophageal reflux; Helicobacter pylori; Peptic ulcer; Potassium; Proton pump inhibitors

\section{Introduction}

Gastric acid secretory inhibitors are effective agents in the treatment of gastroesophageal reflux disease (GERD), peptic ulcer disease, and Helicobacter pylori infection, and in the prevention of low-dose aspirin (LDA) or nonsteroidal anti-inflammatory drug (NSAID)-induced peptic ulcers. ${ }^{1-5}$ Since the market release of proton pump inhibitors (PPIs) in the late 1980s, the severity of the situation regarding gastric acid-related conditions improved dramatically. Vonoprazan, a first-in-class potassium-competitive acid blocker (P-CAB) made in Japan, appeared on the market in 2015, and exhibited rapid, strong, and continuous gastric acid suppres- sion. ${ }^{6}$ As new P-CABs are becoming available, the standard treatment for acid-related conditions is changing.

\section{Pharmacology of Proton Pump Inhibitor}

PPIs are a group of drugs whose main action is a pronounced and long-lasting reduction of gastric acid production by inhibiting the $\mathrm{H}^{+} / \mathrm{K}^{+}$-ATPase enzyme system on the surface of parietal cells. ${ }^{7}$ PPIs are prodrugs which are activated by acid and bind covalently to the gastric $\mathrm{H}^{+} / \mathrm{K}^{+}$-ATPase via a disulfide bond. ${ }^{8}$ Omeprazole was the first PPI introduced, followed by lansoprazole, pantoprazole, rabeprazole, and esomeprazole. PPIs have stronger inhibitory effect on gastric acid secretion than histamine $\mathrm{H} 2$ receptor antago-

\section{Received: August 7, 2018 Revised: September 27, 2018 Accepted: October 25, 2018}

() This is an Open Access article distributed under the terms of the Creative Commons Attribution Non-Commercial License (http://creativecommons. org/licenses/by-nc/4.0) which permits unrestricted non-commercial use, distribution, and reproduction in any medium, provided the original work is properly cited.

*Correspondence: Hidekazu Suzuki, MD, PhD, FACG, AGAF, RFF Fellowship Training Center and Medical Education Center, Keio University School of Medicine, 35 Shinanomachi, Shinjuku-ku, Tokyo 160-8582, Japan Tel: +81-3-5363-3914, Fax: +81-3-5363-3967, E-mail: hsuzuki1201@gmail.com 
nists (H2RAs). ${ }^{7}$ PPIs are metabolized and inactivated by a number of cytochrome P450 (CYP) enzymes (CYP2C9, CYP2C8, CYP2C18, CYP2C19, CYPA3/4, CYP3A1A2, and CYP3A4), mainly CYP2C19 and CYP3A4. ${ }^{9}$ The distribution of CYP2C19 phenotypes was divided into 4 phenotypes: extensive metabolizers (EM), intermediate metabolizers, ultra-rapid metabolizers, and poor metabolizers. ${ }^{10} \mathrm{CYP} 2 \mathrm{C} 19$ is responsible for $>80 \%$ of the metabolism of omeprazole, lansoprazole, and pantoprazole metabolism. ${ }^{11}$ Esomeprazole, the S-isomer of omeprazole, is metabolized to a less extent by CYP2C19 than omeprazole. ${ }^{12}$ Rabeprazole is mainly metabolized via a non-enzymatic reduction to rabeprazole thioether. ${ }^{9}$ Therefore, the effectiveness of omeprazole and lansoprazole is sometimes insufficient in EM and ultra-rapid metabolizers. ${ }^{13}$ Another issue with PPIs is that the effect on gastric acid secretion is slow and reaches a plateau in 3-5 days because several doses are required to inhibit newly synthesized proton pumps and achieve maximal acid-inhibition. ${ }^{14-16}$

\section{Pharmacology of Potassium-competitive Acid Blockers}

$\mathrm{P}-\mathrm{CAB}$ is a class of drug that competitively blocks the potassium-binding site of the gastric $\mathrm{H}^{+} / \mathrm{K}^{+}$-ATPase. ${ }^{6}$ The first $\mathrm{P}-\mathrm{CAB}$ used in clinical practice was revaprazan, which has been available in South Korea and India since 2007. Revaprazan showed an early effect on acid suppression. ${ }^{17}$ However, the effect was not superior to conventional PPIs for the treatment of endoscopic submucosal dissection-induced ulcers ${ }^{18}$ and there are no reports that revaprazan is more effective than PPIs for the other gastric acid-related conditions. In 2015, vonoprazan became the second P-CAB available in the Japanese market, and it is now available in the Philippines, Singapore, and Thailand. Tegoprazan was approved as a treatment for GERD in South Korea since July 2018. Other P-CABs (YH4808, DWP14012, and KFP-H008) are still in clinical trials. ${ }^{19-23}$

Vonoprazan was significantly effective in the treatment of $H$. pylori infection, GERD, and LDA-induced peptic ulcers. ${ }^{6,24}$ There are several advantages to using vonoprazan than conventional PPIs from a pharmacological point of view. It is rapidly absorbed in the small intestine where it accumulated in the canalicular membranes of parietal cells ${ }^{25}$ and it shows a greater acid-inhibitory effect than those of esomeprazole and rabeprazole, as observed from the first day of administration. ${ }^{26}$ It also has a longer duration of action. The $\mathrm{pH} 4$ holding time ratios of vonoprazan were significantly longer than those of esomeprazole and rabeprazole. ${ }^{26}$ It does not require acid activation, whereas PPIs are acid-activated prodrugs that re- quire acid protection. In addition, vonoprazan has a higher value of alkaline $\mathrm{pKa}(>9)$ than PPIs, and previous P-CABs can be easily protonated and accumulated at high concentrations in the intracellular canaliculi of parietal cells. ${ }^{6,8}$ As such, vonoprazan is more stable in an acidic environment than PPIs. ${ }^{27}$ In addition, vonoprazan is shown to have consistent acid suppression capabilities irrespective of CYP2C19. It is metabolized to its inactive form mainly by CYP3A4, and partially also by CYP2B6, CYP2C19, CYP2D6, and SULT2A1, whereas most PPIs are mainly metabolized by CY$\mathrm{P} 2 \mathrm{C} 19{ }^{28}$ Therefore, vonoprazan showed quite rapid, strong, and continuous gastric acid suppression when compared to conventional PPIs. Tegoprazan is another potent and highly selective inhibitor of gastric $\mathrm{H}^{+} / \mathrm{K}^{+}$-ATPase. Interestingly, tegoprazan evoked a gastric phase III contraction of the migrating motor complex in a canine model. ${ }^{19}$ YH4808 had a faster onset than esomeprazole and can maintain an intra-gastric acidity of $\mathrm{pH}>4$ for a longer time during both day and night in healthy volunteers. ${ }^{20}$ DWP14012 showed rapid and sustained suppression of gastric acid secretion in healthy volunteers. ${ }^{22}$ KFP-H008 showed a more effective, potent, and longer-lasting inhibitory action than lansoprazole in a rat model. ${ }^{23}$ However, there are no reports about the usefulness of tegoprazan, YH4808, DWP14012, and KFP-H008 in actual clinical practice.

\section{Gastroesophageal Reflux Disease}

For most patients with GERD, PPIs are the first choice of treatment. ${ }^{24} \mathrm{~A}$ recent Japanese guideline for GERD recommended the use of PPIs as first-line and maintenance treatments (Fig. 1A). ${ }^{29}$ PPIs were shown to be superior to H2RAs in healing erosive esophagitis and decreasing relapse rates. ${ }^{30-32} \mathrm{~A}$ randomized study suggested that esomeprazole has a more rapid effect against heartburn and reflux symptoms than omeprazole, lansoprazole, and pantoprazole; however, there was no significant difference in the rate of endoscopic healing of reflux esophagitis at 8 weeks. ${ }^{33}$ Despite this, most studies determined that there is no notable difference in effect among PPIs in terms of short-term symptom management. ${ }^{34}$

Vonoprazan could be the first-line drug in the future. In CYP2C19 EM patients with erosive esophagitis, 90.0\% treated with vonoprazan $(20 \mathrm{mg} /$ day $)$ achieved mucosal healing at 2 weeks compared with the $79.3 \%$ that were treated with lansoprazole $(P<$ $0.01){ }^{35}$ Similarly, at 4 and 8 weeks, the proportion of patients with healed erosive esophagitis tended to be higher in the vonoprazan group than in the lansoprazole group (at 4 weeks $96.1 \%$ vs $90.9 \%$, $P<0.05$; at 8 weeks $98.9 \%$ vs $94.5 \%, P<0.03) .{ }^{35}$ Moreover, vonoprazan was more effective for severe erosive esophagitis (Los 

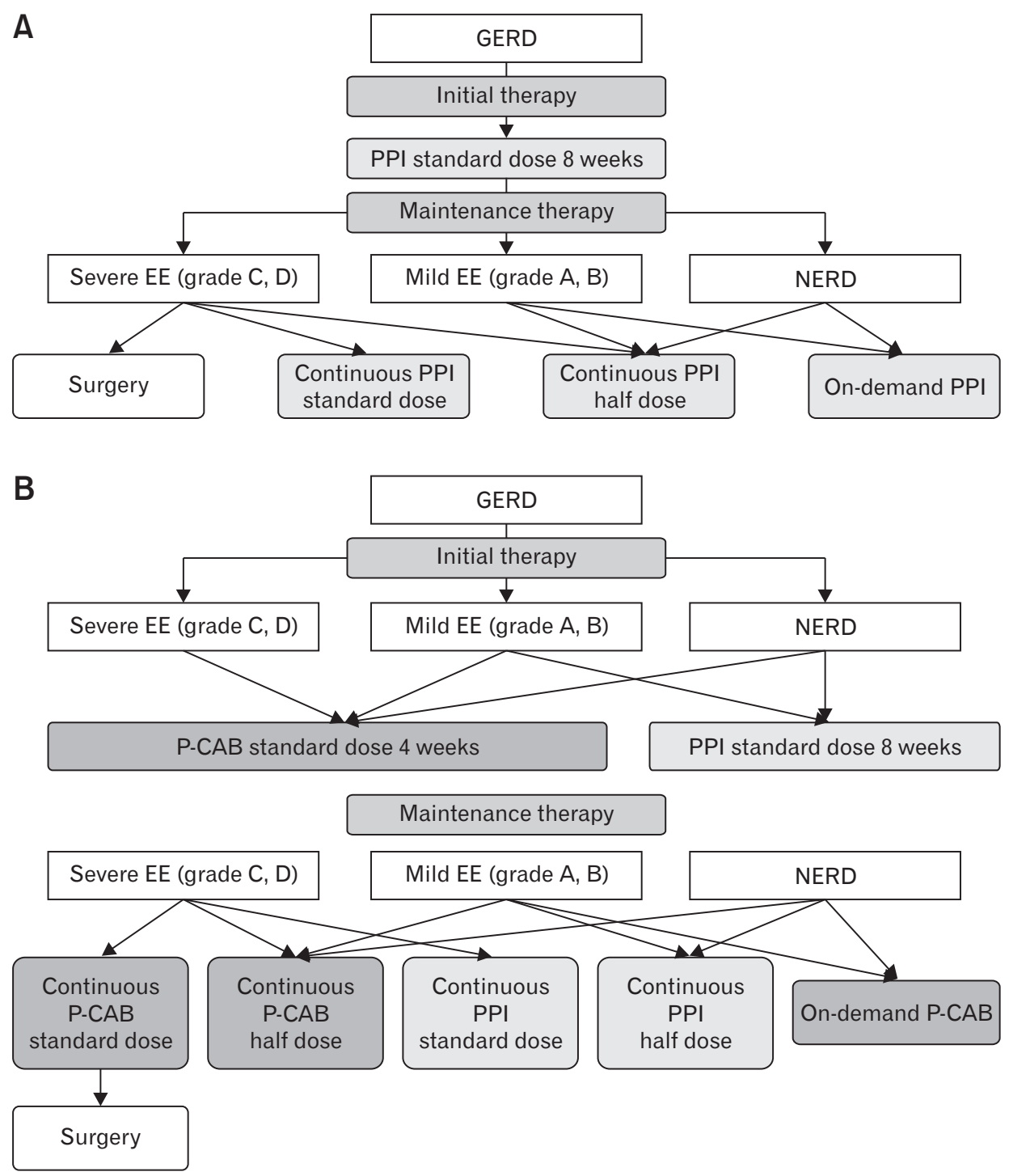

Figure 1. Strategy of gastroesophageal reflux disease (GERD) treatment. (A) Conventional strategy of GERD treatment (an abridged edition of the Evidence-based Clinical Practice Guidelines for GERD 2015 published by the Japanese Society of Gastroenterology). ${ }^{29}$ Initial treatment is administration of proton pump inhibitor (PPI) standard dose for 8 weeks. Then, maintenance treatment is administration of PPI half dose daily. If incomplete healing occurs, maintenance with continuous PPI standard dose is permissible. On-demand PPI treatment is alternative management strategy. (B) The authors propose a new strategy of GERD treatment considering the effectiveness of potassium-competitive acid blocker (P$\mathrm{CAB}$ ). Initial treatment is 4-week treatment with $\mathrm{P}-\mathrm{CAB}$ standard dose for severe erosive esophagitis. Four-week treatment with $\mathrm{P}-\mathrm{CAB}$ or 8 -week treatment with PPI are recommended as an initial therapy for mild erosive esophagitis or non-erosive reflux disease (NERD). Then, maintenance treatment is administration of $\mathrm{P}-\mathrm{CAB}$ half dose daily. If incomplete healing, maintenance with continuous $\mathrm{P}-\mathrm{CAB}$ standard dose is permissible. Continuous PPI standard or half dose daily is one of the options. On-demand P-CAB treatment is a workable alternative management strategy. The severity of reflux esophagitis is classified according to the Los Angeles classification. EE, erosive esophagitis.

Angeles classification grades C/D) than lansoprazole (at 8 weeks $98.7 \%$ vs $87.5 \%, P<0.01$ ) and had more rapid effectiveness (at 2 weeks $88.0 \%$ vs $63.9 \%, \mathrm{P}<0.01$; at 4 weeks $96.0 \%$ vs $80.6 \%$, $P<0.03) .^{35}$ Therefore, vonoprazan is highly effective for patients with more severe erosive esophagitis and CYP2C19 EM patients.
Even in whole patients, vonoprazan is more effective than lansoprazole at each time point (at 2 weeks $90.7 \%$ vs $81.9 \%, P<0.01$; at 4 weeks $96.6 \%$ vs $92.5 \%, P<0.01$; at 8 weeks $99.0 \%$ vs $95.5 \%$, $P<0.01)$. On the other contrary, no data showed that $\mathrm{P}-\mathrm{CAB}$ was effective to relieve the symptoms of mild esophagitis and NERD. 
A non-randomized, controlled trial reported that 4-week treatment with vonoprazan $(20 \mathrm{mg} /$ day) was effective to relieve symptoms in $66.7 \%$ of NERD patients and $53.8 \%$ of PPI-resistant NERD patients. ${ }^{36}$ In summary, 4-week treatment with vonoprazan $(20 \mathrm{mg} /$ day) was effective as an initial therapy for severe erosive esophagitis (Fig. 1B), and 4-week treatment with vonoprazan and 8-week treatment with a PPI were recommended as initial therapies for mild erosive esophagitis and NERD (Fig. 1B). Conventionally, double dose PPI therapy, combination therapy of PPI and H2RA and the addition of a prokinetic agent to PPI were used for PPIresistant reflux esophagitis (Fig. 2A). ${ }^{37-40}$ Vonoprazan seems to be effective for PPI-resistant reflux esophagitis. ${ }^{41}$ Twenty-four PPIresistant reflux esophagitis patients were enrolled in this study and $58.3 \%$ had severe erosive esophagitis. In total, $87.5 \%$ of the whole patients and $85.7 \%$ of severe erosive esophagitis patients achieved endoscopic healing of erosive esophagitis at 4 weeks after drug change from PPI to vonoprazan $(20 \mathrm{mg} / \mathrm{day}) .{ }^{41}$ In addition, the symptoms of GERD were significantly improved from the day after drug change. ${ }^{41}$ Vonoprazan (40 mg/day) rescue therapy may also be useful for PPI-resistant reflux esophagitis. ${ }^{42}$ Shinozaki et $\mathrm{al}^{43}$ reported that vonoprazan $(10 \mathrm{mg} /$ day; maintenance dose $)$ treatment is also effective for PPI-resistant reflux esophagitis. Thus, we believe that the first choice for the treatment of PPI-resistant reflux esophagitis will be vonoprazan in the near future (Fig. 2B). There is no evidence of $\mathrm{P}-\mathrm{CAB}$ plus prokinetic combination treatment for PPI-resistant reflux esophagitis until now. Further study is needed to additional effect of prokinetics on P-CAB based treatment.

Continuous administration of vonoprazan $(10 \mathrm{mg} / \mathrm{day}$ or 20 $\mathrm{mg} /$ day) is more efficacious than that of lansoprazole (15 mg/day) in maintaining healed erosive esophagitis. ${ }^{44}$ Therefore, at present, the optimal maintenance strategy for erosive esophagitis is continuous administration of vonoprazan at $10 \mathrm{mg} /$ day (Fig. 1B). In addition, on-demand PPI therapy is an attractive option for long-term management of GERD. However, because of the slow onset of action of PPIs, their efficacy is sometimes insufficient. ${ }^{45}$ Umezawa et $\mathrm{al}^{46}$ showed that on-demand therapy using vonoprazan is more effective than continuous PPI treatment as an alternative maintenance therapy for patients with mild erosive esophagitis. These data could change the strategy of GERD treatment around the world (Fig. 1B). Comparative clinical studies are being conducted on an every-other-day administration of vonoprazan $(20 \mathrm{mg} /$ day $)$ and lansoprazole $(15 \mathrm{mg} /$ day $)$ as maintenance treatments for erosive esophagitis. ${ }^{47}$ There is little evidence on other P-CABs being used to successfully treat GERD. A phase-III clinical trial on reflux esophagitis is currently being conducted in Korea to compare the

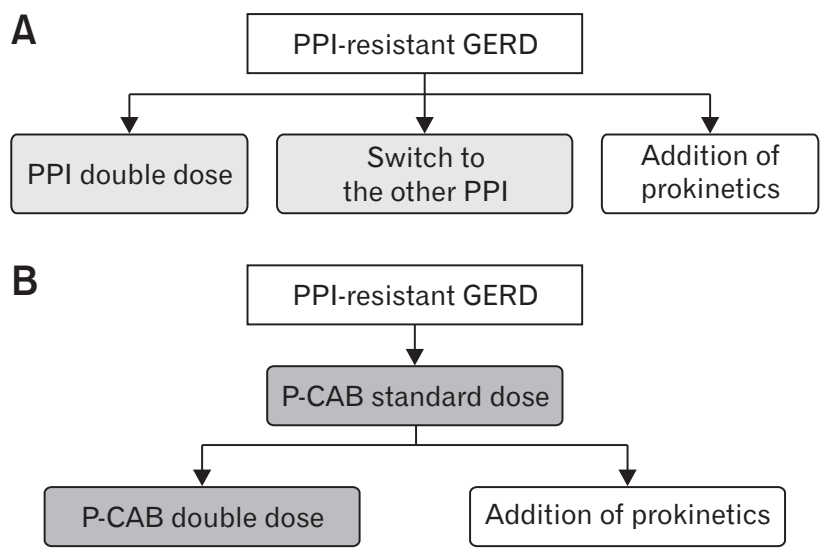

Figure 2. Strategy of proton pump inhibitor (PPI)-resistant gastroesophageal reflux disease (GERD) treatment. (A) Conventional strategy of PPI-resistant GERD treatment (an abridged edition of the Evidence-based Clinical Practice Guidelines for GERD 2015 published by the Japanese Society of Gastroenterology). ${ }^{29}$ First choice is double dose PPI therapy. Switching to the other PPI is an option. Addition of prokinetics to PPI is effective in some cases. (B) The authors propose a new strategy of PPI-resistant GERD treatment. First choice is administration of potassium-competitive acid blocker $(\mathrm{P}$ $\mathrm{CAB}$ ) standard dose. Addition of prokinetics to $\mathrm{P}-\mathrm{CAB}$ may have an additional effect.

safety and efficacy of a new P-CAB, tegoprazan (50 mg and 100 $\mathrm{mg})$, with those of esomeprazole $40 \mathrm{mg}^{6}$

\section{Helicobacter pylori Eradication}

Induction of a strong acid inhibition stimulates growth of $H$. pylori and increases the bactericidal effect of amoxicillin, hence, PPIs are important for $H$. pylori eradication. ${ }^{48}$ A meta-analysis revealed that high-dose PPIs are more effective than standarddose for curing an $H$. pylori infection. ${ }^{49} \mathrm{CYP} 2 \mathrm{C} 19$ polymorphisms are associated with the efficacy of PPI-based $H$. pylori eradication therapy. The eradication rates of triple omeprazole and lansoprazole therapies were lower in EM than in the other groups. ${ }^{50,51}$ Eradication regimens involving esomeprazole, rabeprazole, and new generation PPIs showed better overall $H$. pylori eradication rates (especially those in CYP2C19 EM patients) than those involving omeprazole, lansoprazole, and pantoprazole. ${ }^{52}$

Vonoprazan-amoxicillin-clarithromycin triple regimen dramatically improved the eradication rate of first-line $H$. pylori treatment. The eradication rate was $92.6 \%$ with vonoprazan-based triple therapy versus $75.9 \%$ with lansoprazole-based triple therapy. ${ }^{53}$ The eradication rate was significantly higher with vonoprazan compared with lansoprazole in those patients infected with clarithromycin- 
resistant strains $(82.0 \%$ vs $40.0 \%, P<0.01) .{ }^{53}$ In CYP2C19 EM patients, the eradication rate of vonoprazan-based triple therapy was significantly higher than that of lansoprazole-based triple therapy (92.9\% vs $75.0 \%, P<0.01) .{ }^{53}$ In CYP2C19 PM patients, there was no significant difference, but the rate of vonoprazan-based triple therapy was high compared with the rate of lansoprazole-based triple therapy ( $90.9 \%$ vs $81.3 \%, P=\mathrm{NS}$ ). Conversely, the rate of the second-line metronidazole and amoxicillin-based triple therapy did not differ significantly between the PPI and vonoprazan groups (96.8\% vs $90.5 \%, P=\mathrm{NS}) .^{54}$ A meta-analysis showed that vonoprazan- and conventional PPI-based therapies are similarly effective in eradicating clarithromycin-susceptible $H$. pylori strains $(95.4 \%$ vs $92.8 \%, P=0.230$ ), and that vonoprazan-based triple therapy was significantly superior to PPI-based therapy in treating patients infected with clarithromycin-resistant $H$. pylori strains $(82.0 \%$ vs $40.0 \%, P<0.01) .{ }^{55}$ Ono et $\mathrm{al}^{56}$ showed that a clarithromycin-metronidazole-vonoprazan regimen had greater efficacy than clarithromycin-metronidazole-PPI regimen for penicillin allergy patients (92.9\% vs $54.6 \%, P<0.01$ ). There is geographic distribution of resistance to clarithromycin and metronidazole in $H$. pylori. High resistance to clarithromycin and low resistance to metronidazole are found in Japan. ${ }^{57}$ Further studies are needed to evaluate the efficacy of vonoprazan-based therapy because vonoprazan has been approved only in Japan, Philippines, Singapore, and Thailand at this time. The Maastricht V/Florence Consensus Report recommended that the optimal regimen should be selected with the prevalence of antibiotic resistance rates (Fig. 3A). ${ }^{58}$ Amoxicillin-clarithromycinPPI triple therapy without prior susceptibility testing should be abandoned when the clarithromycin resistance rate in the region is more than $15 \% .^{58}$ In the area with high resistance to clarithromycin and low resistance to metronidazole, amoxicillin-metronidazole-PPI triple regimen is recommended. ${ }^{58}$ In the area with dual resistance to clarithromycin and metronidazole, bismuth quadruple therapy is recommended. As a rescue treatment, bismuth quadruple therapy or quinolone-containing therapy is recommended. ${ }^{58}$ In the area with low resistance to clarithromycin, we recommend the amoxicillinclarithromycin-vonoprazan triple regimen. If antimicrobial susceptibility testing is available, amoxicillin-clarithromycin-PPI triple regimen can be an alternative choice. In the area with high resistance to clarithromycin and low resistance to metronidazole, amoxicillinmetronidazole-vonoprazan or PPI triple regimen is recommended because there is no evidence of the amoxicillin-metronidazolevonoprazan triple regimen being more effective than the amoxicillin-metronidazole-PPI triple regimen. Little has been reported on bismuth quadruple therapy, quinolone-containing therapy, and vo- noprazan (or other P-CABs)-based concomitant therapy; therefore, studies on this regard are expected (Fig. 3B). Currently, a phase III clinical trial for $H$. pylori eradication is being conducted in Korea to compare tegoprazan-amoxicillin-clarithromycin triple regimen with lansoprazole-amoxicillin-clarithromycin triple regimen.

\section{Low-dose Aspirin or Nonsteroidal Anti-inflammatory Drug-induced Peptic Ulcers}

The use of LDA in the prevention of atherothrombosis is frequently associated with a development of erosions or ulcerations in the upper gastrointestinal tract. Compared to controls, PPIs reduced the risk of LDA-induced peptic ulcers but did not increase the risk of mortality. ${ }^{59,60}$ Another meta-analysis showed that PPIs were superior to H2RAs for prevention of LDA-induced peptic ulcers. ${ }^{5}$ PPIs are also effective and safe in preventing NSAIDinduced peptic ulcers when compared to placebos and H2RAs. ${ }^{61,62}$ Recently, vonoprazan showed an equivalent efficacy to that of lansoprazole in preventing LDA-induced ulcer recurrence (Farrington and Manning test: margin 8.7\%, significance level 2.5\%). At the same time, peptic ulcer recurrence rates were significantly lower with vonoprazan $10 \mathrm{mg}$ than with lansoprazole $15 \mathrm{mg}$, as shown by the results of the post hoc analyses of the extension study (log-rank test, $P=0.039){ }^{63}$ The 24 -week peptic ulcer recurrence rate was $2.8 \%$ and $0.5 \%$ in the lansoprazole $(15 \mathrm{mg})$ and vonoprazan $(10 \mathrm{mg})$ groups, respectively. With regard to NSAID-induced peptic ulcers, vonoprazan showed better efficacy. ${ }^{64}$ The proportion of patients with endoscopically confirmed recurrent NSAID-induced peptic ulcers within 24 weeks was $3.3 \%, 3.4 \%$, and $5.5 \%$, for vonoprazan (10 mg, $20 \mathrm{mg}$ ) and lansoprazole (15 mg), respectively. ${ }^{64}$

\section{Complications}

The long-term use of PPIs could cause the development of fundic gland polyps, carcinoid tumors, increase the risk of community acquired pneumonia, iron and vitamin B12 deficiencies, Clostridium difficile-associated diarrhea, dementia, and chronic kidney disease. ${ }^{25,65-72}$ Clinical trials evaluating the safety and tolerability of vonoprazan revealed that most of treatment-emergent adverse events with vonoprazan were mild in intensity and there was no obvious trend towards an increase in incidence over time up to 52 weeks of treatment. ${ }^{35,63}$ In these trials, hepatotoxicity was rarely observed. One differential characteristic of vonoprazan is that it is a pyrrole derivate, whereas previous $\mathrm{P}-\mathrm{CABs}$ are imidazole-pyridine 

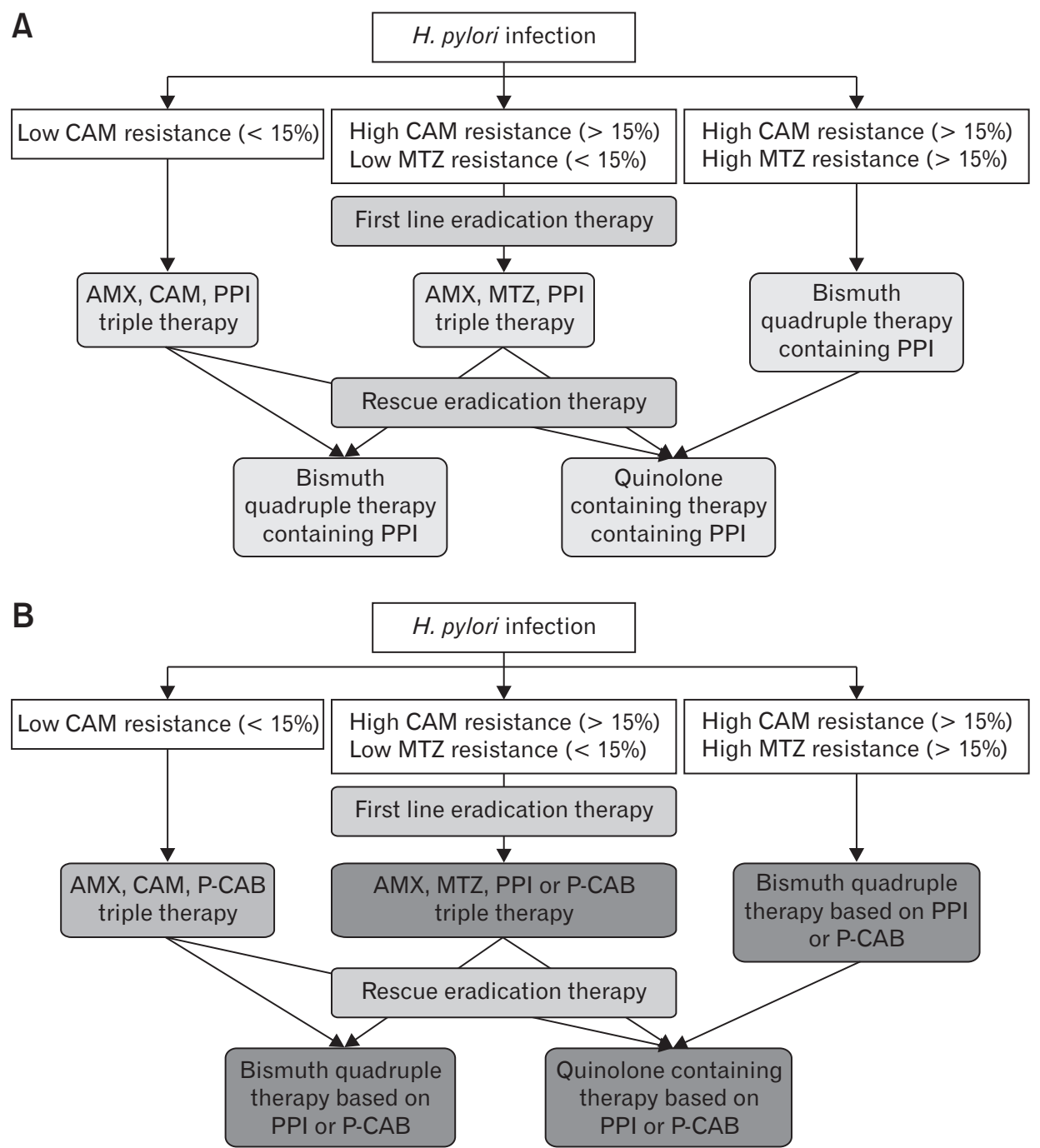

Figure 3. Strategy of proton pump inhibitor (PPI)-resistant Helicobacter pylori (H. pylori) eradication treatment. (A) Conventional strategy of $H$. pylori eradication treatment (an abridged edition of Management of Helicobacter pylori infection-the Maastricht V/Florence Consensus Report published by the European Helicobacter and Microbiota Study Group). ${ }^{58}$ In areas of low clarithromycin $(\mathrm{CAM})$ resistance $(<15 \%)$, amoxicillin (AMX), CAM, and PPI triple therapy is the recommended first-line treatment. In areas of high CAM resistance $(>15 \%)$ and low metronidazole (MTZ) resistance $(<15 \%)$, AMX, MTZ, and PPI triple therapy is recommended. In areas of dual resistance, bismuth quadruple therapy is recommended. Quinolone containing therapy and bismuth quadruple therapy are recommended as rescue therapy. (B) The authors propose a new strategy of $H$. pylori eradication treatment. AMX, CAM, and potassium-competitive acid blocker (P-CAB) triple therapy is sufficient treatment even for CAM resistant strains. AMX, MTZ, P-CAB, or PPI triple therapy, quinolone containing therapy and bismuth quadruple therapy may be recommended as rescue therapy.

derivates, which might cause the hepatotoxicity of previous P-CABs (SCH 28080 and AZD 0865) and subsequent termination of their clinical development. ${ }^{73,74}$ Serum gastrin levels tended to be higher in the vonoprazan group than in the PPI group. ${ }^{75,76}$ Although there is no evidence that hypergastrinemia induced by PPIs or PCABs causes neuroendocrine tumors in humans, P-CAB-induced stimulation of enterochromaffin cells and increased risk of neuroen- docrine tumors remained. Bone fracture, hypomagnesemia, pneumonia, and $C$. difficile-associated diarrhea may also be caused by strong inhibition of gastric acid by continuous P-CABs treatment. ${ }^{6}$ In addition, parietal cell protrusion and dilated oxyntic glands can be observed after vonoprazan treatment. ${ }^{77}$ Vonoprazan treatment reduced the value of urea breath test in patients infected with $H$. pylori. $^{78}$ The long-term (5 years) effect of vonoprazan is currently 
under examination in patients receiving maintenance treatment for healed erosive esophagitis, and complications of long-term vonoprazan use need to be re-evaluated in the future.

\section{Conclusions}

Vonoprazan, an effective P-CAB, improves the treatment of gastric acid-related conditions including GERD, $H$. pylori infection and LDA/NSAID-induced peptic ulcers. It is highly likely that global standard therapy for gastric acid-related conditions will change in the future. The long-term safety of vonoprazan is still being investigated. Because studies on other P-CABs are limited, further research is required to show the superiority of other P-CABs to conventional PPIs.

Acknowledgements: The part of this article has been presented in the lecture at Asian Postgraduate Course on Neurogastroenterology and Motility 2018, Seoul, Korea by Hidekazu Suzuki.

Financial support: This work was supported by a Grant-in-Aid for Scientific Research B (16H05291) to Hidekazu Suzuki from the Japan Society for the Promotion of Science.

Conflicts of interest: Hidekazu Suzuki received scholarship funds for the research from Daiichi-Sankyo, EA Pharma, Otsuka Pharmaceutical, and Tsumura, and received service honoraria from Astellas, AstraZeneca, Daiichi-Sankyo, Otsuka Pharmaceutical, Mylan EPD, Takeda Pharmaceutical, and Tsumura.

Author contributions: Hideki Mori and Hidekazu Suzuki wrote the manuscript; Hideki Mori made the Figures; and Hidekazu Suzuki supervised the all preparation of this article.

\section{References}

1. Mori H, Suzuki H, Matsuzaki J, et al. Efficacy of 10-day sitafloxacincontaining third-line rescue therapies for Helicobacter pylori strains containing the gyrA mutation. Helicobacter 2016;21:286-294.

2. Mori H, Suzuki H, Matsuzaki J, et al. Rifabutin-based 10-day and 14-day triple therapy as a third-line and fourth-line regimen for Helicobacter pylori eradication: a pilot study. United European Gastroenterol J 2016;4:380-387.

3. Gyawali CP, Fass R. Management of gastroesophageal reflux disease. Gastroenterology 2018;154:302-318.

4. Tajima A, Koizumi K, Suzuki K, et al. Proton pump inhibitors and recurrent bleeding in peptic ulcer disease. J Gastroenterol Hepatol 2008;23(suppl 2):S237-S241.
5. Mo C, Sun G, Wang YZ, Lu ML, Yang YS. PPI versus histamine H2 receptor antagonists for prevention of upper gastrointestinal injury associated with low-dose aspirin: systematic review and meta-analysis. PLoS One 2015;10:e0131558.

6. Sugano K. Vonoprazan fumarate, a novel potassium-competitive acid blocker, in the management of gastroesophageal reflux disease: safety and clinical evidence to date. Therap Adv Gastroenterol 2018;11:1756283X17745776.

7. Huang JQ, Hunt RH. Pharmacological and pharmacodynamic essentials of $\mathrm{H}_{2}$-receptor antagonists and proton pump inhibitors for the practising physician. Best Pract Res Clin Gastroenterol 2001;15:355-370.

8. Shin JM, Kim N. Pharmacokinetics and pharmacodynamics of the proton pump inhibitors. J Neurogastroenterol Motil 2013;19:25-35.

9. Ishizaki T, Horai Y. Review article: cytochrome P450 and the metabolism of proton pump inhibitors--emphasis on rabeprazole. Aliment Pharmacol Ther 1999;13(suppl 3):27-36.

10. Deshpande N, V S, V V RK, et al. Rapid and ultra-rapid metabolizers with $C Y P 2 C 19 * 17$ polymorphism do not respond to standard therapy with proton pump inhibitors. Meta Gene 2016;9:159-164.

11. Andersson T, Holmberg J, Röhss K, Walan A. Pharmacokinetics and effect on caffeine metabolism of the proton pump inhibitors, omeprazole, lansoprazole, and pantoprazole. Br J Clin Pharmacol 1998;45:369-375.

12. Abelö A, Andersson TB, Antonsson M, Naudot AK, Skånberg I, Weidolf L. Stereoselective metabolism of omeprazole by human cytochrome P450 enzymes. Drug Metab Dispos 2000;28:966-972.

13. Kuo $\mathrm{CH}$, Lu CY, Shih HY, et al. CYP2C19 polymorphism influences Helicobacter pylori eradication. World J Gastroenterol 2014;20:1602916036.

14. Junghard O, Hassan-Alin M, Hasselgren G. The effect of the area under the plasma concentration vs time curve and the maximum plasma concentration of esomeprazole on intragastric $\mathrm{pH}$. Eur J Clin Pharmacol 2002;58:453-458.

15. Mejia A, Kraft WK. Acid peptic diseases: pharmacological approach to treatment. Expert Rev Clin Pharmacol 2009;2:295-314.

16. Sachs G, Shin JM, Munson K, et al. Review article: the control of gastric acid and Helicobacter pylori eradication. Aliment Pharmacol Ther 2000;14:1383-1401.

17. Yu KS, Bae KS, Shon JH, et al. Pharmacokinetic and pharmacodynamic evaluation of a novel proton pump inhibitor, YH1885, in healthy volunteers. J Clin Pharmacol 2004;44:73-82.

18. Kim YG, Jang BI, Kim TN. A matched case-control study of a novel acid-pump antagonist and proton-pump inhibitor for the treatment of iatrogenic ulcers caused by endoscopic submucosal dissection. Gut and liver 2010;4:25-30.

19. Takahashi N, Take Y. Tegoprazan, a novel potassium-competitive acid blocker to control gastric acid secretion and motility. J Pharmacol Exp Ther 2018;364:275-286.

20. Yi S, Lee $\mathrm{H}$, Jang SB, et al. A novel $\mathrm{K}^{+}$competitive acid blocker, YH4808, sustains inhibition of gastric acid secretion with a faster onset than esomeprazole: randomised clinical study in healthy volunteers. Aliment Pharmacol Ther 2017;46:337-346.

21. Kim E, Kim A, Yi S, et al. Effect of food on the pharmacokinetics of 
YH4808, a potassium-competitive acid blocker, after single- and multiple-oral dosing in healthy subjects. Eur J Clin Pharmacol 2018;74:12611272.

22. Sunwoo J, Oh J, Moon SJ, et al. Safety, tolerability, pharmacodynamics and pharmacokinetics of DWP14012, a novel potassium-competitive acid blocker, in healthy male subjects. Aliment Pharmacol Ther 2018;48:206-218.

23. Li CY, Su M, Yan YY, et al. KFP-H008 blocks gastric acid secretion through inhibiting $\mathrm{H}^{+}-\mathrm{K}^{+}$-ATPase. Eur J Pharmacol 2017;810:112119.

24. Oshima T, Miwa H. Potent potassium-competitive acid blockers: a new era for the treatment of acid-related diseases. J Neurogastroenterol Motil 2018;30:334-344.

25. Klatte DCF, Gasparini A, Xu H, et al. Association between proton pump inhibitor use and risk of progression of chronic kidney disease. Gastroenterology 2017;153:702-710.

26. Sakurai Y, Mori Y, Okamoto H, et al. Acid-inhibitory effects of vonoprazan $20 \mathrm{mg}$ compared with esomeprazole $20 \mathrm{mg}$ or rabeprazole $10 \mathrm{mg}$ in healthy adult male subjects--a randomised open-label cross-over study. Aliment Pharmacol Ther 2015;42:719-730.

27. Scott DR, Munson KB, Marcus EA, Lambrecht NW, Sachs G. The binding selectivity of vonoprazan (TAK-438) to the gastric $\mathrm{H}^{+}, \mathrm{K}^{+}$ -ATPase. Aliment Pharmacol Ther 2015;42:1315-1326.

28. Kagami T, Sahara S, Ichikawa H, et al. Potent acid inhibition by vonoprazan in comparison with esomeprazole, with reference to CYP2C19 genotype. Aliment Pharmacol Ther 2016;43:1048-1059.

29. Iwakiri K, Kinoshita Y, Habu Y, et al. Evidence-based clinical practice guidelines for gastroesophageal reflux disease 2015. J Gastroenterol 2016;51:751-767.

30. Chiba N, De Gara CJ, Wilkinson JM, Hunt RH. Speed of healing and symptom relief in grade II to IV gastroesophageal reflux disease: a metaanalysis. Gastroenterology 1997;112:1798-1810.

31. Caro JJ, Salas M, Ward A. Healing and relapse rates in gastroesophageal reflux disease treated with the newer proton-pump inhibitors lansoprazole, rabeprazole, and pantoprazole compared with omeprazole, ranitidine, and placebo: evidence from randomized clinical trials. Clin Ther 2001;23:998-1017.

32. Kawano S, Murata H, Tsuji S, et al. Randomized comparative study of omeprazole and famotidine in reflux esophagitis. J Gastroenterol Hepatol 2002;17:955-959.

33. Zheng RN. Comparative study of omeprazole, lansoprazole, pantoprazole and esomeprazole for symptom relief in patients with reflux esophagitis. World J Gastroenterol 2009; 15:990-995.

34. Khan M, Santana J, Donnellan C, Preston C, Moayyedi P. Medical treatments in the short term management of reflux oesophagitis. Cochrane Database Syst Rev 2007:CD003244.

35. Ashida K, Sakurai Y, Hori T, et al. Randomised clinical trial: vonoprazan, a novel potassium-competitive acid blocker, vs. lansoprazole for the healing of erosive oesophagitis. Aliment Pharmacol Ther 2016;43:240-251.

36. Asaoka D, Nagahara A, Hojo M, et al. Efficacy of a potassiumcompetitive acid blocker for improving symptoms in patients with reflux esophagitis, non-erosive reflux disease, and functional dyspepsia. Biomed
Rop 2017;6:175-180.

37. Kinoshita Y, Hongo M. Efficacy of twice-daily rabeprazole for reflux esophagitis patients refractory to standard once-daily administration of PPI: the Japan-based TWICE study. Am J Gastroenterol 2012;107:522530 .

38. Adachi K, Komazawa Y, Mihara T, et al. Administration of $\mathrm{H} 2$ receptor antagonist with proton pump inhibitor is effective for long-term control of refractory reflux esophagitis. J Clin Gastroenterol 2004;38:297-298.

39. Lim HC, Kim JH, Youn YH, Lee EH, Lee BK, Park H. Effects of the addition of mosapride to gastroesophageal reflux disease patients on proton pump inhibitor: a prospective randomized, double-blind study. J Neurogastroenterol Motil 2013;19:495-502.

40. Takeuchi T, Takahashi Y, Kawaguchi S, et al. Therapy of gastroesophageal reflux disease and functional dyspepsia overlaps with symptoms after usual-dose proton pump inhibitor: acotiamide plus usual-dose proton pump inhibitor versus double-dose proton pump inhibitor. J Gastroenterol Hepatol 2018;33:623-630.

41. Hoshino S, Kawami N, Takenouchi N, et al. Efficacy of vonoprazan for proton pump inhibitor-resistant reflux ssophagitis. Digestion 2017;95:156-161.

42. Iwakiri K, Sakurai Y, Shiino M, et al. A randomized, double-blind study to evaluate the acid-inhibitory effect of vonoprazan $(20 \mathrm{mg}$ and $40 \mathrm{mg}$ ) in patients with proton-pump inhibitor-resistant erosive esophagitis. Therap Adv Gastroenterol 2017;10:439-451.

43. Shinozaki S, Osawa H, Hayashi Y, et al. Vonoprazan $10 \mathrm{mg}$ daily is effective for the treatment of patients with proton pump inhibitor-resistant gastroesophageal reflux disease. Biomed Rep 2017;7:231-235.

44. Ashida K, Iwakiri K, Hiramatsu N, et al. Maintenance for healed erosive esophagitis: phase III comparison of vonoprazan with lansoprazole. World J Gastroenterol 2018;24:1550-1561.

45. Leodolter A, Penagini R. On-demand therapy is a valid strategy in GERD patients: pros and cons. Dig Dis 2007;25:175-178.

46. Umezawa M, Kawami N, Hoshino S, et al. Efficacy of on-demand therapy using 20-mg vonoprazan for mild reflux esophagitis. Digestion 2018;97:309-315.

47. Kato M, Ito N, Demura M, Kubo K, Mabe K, Harada N. Study for every other day administration of vonoprazan in maintenance treatment of erosive GERD: study protocol for a multicentre randomised cross-over study. BMJ Open Gastroenterol 2018;5:e000197.

48. Marcus EA, Inatomi N, Nagami GT, Sachs G, Scott DR. The effects of varying acidity on Helicobacter pylori growth and the bactericidal efficacy of ampicillin. Aliment Pharmacol Ther 2012;36:972-979.

49. Villoria A, Garcia P, Calvet X, Gisbert JP, Vergara M. Meta-analysis: high-dose proton pump inhibitors vs. standard dose in triple therapy for Helicobacter pylori eradication. Aliment Pharmacol Ther 2008;28:868877.

50. Zhao F, Wang J, Yang Y, et al. Effect of CYP2C19 genetic polymorphisms on the efficacy of proton pump inhibitor-based triple therapy for Helicobacter pylori eradication: a meta-analysis. Helicobacter 2008;13:532-541.

51. Furuta T, Shirai N, Sugimoto M, Nakamura A, Hishida A, Ishizaki T. Influence of CYP2C19 pharmacogenetic polymorphism on proton pump 
inhibitor-based therapies. Drug Metab Pharmacokinet 2005;20:153167.

52. McNicholl AG, Linares PM, Nyssen OP, Calvet X, Gisbert JP. Metaanalysis: esomeprazole or rabeprazole vs. first-generation pump inhibitors in the treatment of Helicobacter pylori infection. Aliment Pharmacol Ther 2012;36:414-425.

53. Murakami K, Sakurai Y, Shiino M, Funao N, Nishimura A, Asaka M. Vonoprazan, a novel potassium-competitive acid blocker, as a component of first-line and second-line triple therapy for Helicobacter pylori eradication: a phase III, randomised, double-blind study. Gut 2016;65:14391446.

54. Nishizawa T, Suzuki H, Fujimoto A, et al. Effects of patient age and choice of antisecretory agent on success of eradication therapy for Helicobacter pylori infection. J Clin Biochem Nutr 2017;60:208-210.

55. Li M, Oshima T, Horikawa T, et al. Systematic review with meta-analysis: vonoprazan, a potent acid blocker, is superior to proton-pump inhibitors for eradication of clarithromycin-resistant strains of Helicobacter pylori. Helicobacter 2018;23:e12495.

56. Ono S, Kato M, Nakagawa S, Mabe K, Sakamoto N. Vonoprazan improves the efficacy of Helicobacter pylori eradication therapy with a regimen consisting of clarithromycin and metronidazole in patients allergic to penicillin. Helicobacter 2017;22.

57. Suzuki H, Mori H. World trends for $H$. pylori eradication therapy and gastric cancer prevention strategy by $H$. pylori test-and-treat. J Gastroenterol 2018;53:354-361.

58. Malfertheiner P, Megraud F, O'Morain CA, et al. Management of Helicobacter pylori infection-the Maastricht V/Florence Consensus Report. Gut 2017;66:6-30.

59. Dahal K, Sharma SP, Kaur J, Anderson BJ, Singh G. Efficacy and safety of proton pump inhibitors in the long-term aspirin users: a meta-analysis of randomized controlled trials. Am J Ther 2017;24:e559-e569.

60. Iwakiri R, Higuchi K, Kato M, et al. Randomised clinical trial: prevention of recurrence of peptic ulcers by rabeprazole in patients taking lowdose aspirin. Aliment Pharmacol Ther 2014;40:780-795.

61. Yang M, He M, Zhao M, et al. Proton pump inhibitors for preventing non-steroidal anti-inflammatory drug induced gastrointestinal toxicity: a systematic review. Curr Med Res Opin 2017;33:973-980.

62. Sugano K, Kinoshita Y, Miwa H, Takeuchi T. Randomised clinical trial: esomeprazole for the prevention of nonsteroidal anti-inflammatory drug-related peptic ulcers in Japanese patients. Aliment Pharmacol Ther 2012;36:115-125.

63. Kawai T, Oda K, Funao N, et al. Vonoprazan prevents low-dose aspirin-associated ulcer recurrence: randomised phase 3 study. Gut 2018;67:1033-1041.

64. Mizokami Y, Oda K, Funao N, et al. Vonoprazan prevents ulcer recurrence during long-term NSAID therapy: randomised, lansoprazolecontrolled non-inferiority and single-blind extension study. Gut
2018;67:1042-1051

65. Thomson AB, Sauve MD, Kassam N, Kamitakahara H. Safety of the long-term use of proton pump inhibitors. World J Gastroenterol 2010;16:2323-2330.

66. Otsuka T, Sugimoto M, Inoue R, et al. Influence of potassium-competitive acid blocker on the gut microbiome of Helicobacter pylori-negative healthy individuals. Gut 2017;66:1723-1725.

67. Hongo M, Fujimoto K. Incidence and risk factor of fundic gland polyp and hyperplastic polyp in long-term proton pump inhibitor therapy: a prospective study in Japan. J Gastroenterol 2010;45:618-624.

68. Jianu CS, Fossmark R, Viset T, et al. Gastric carcinoids after long-term use of a proton pump inhibitor. Aliment Pharmacol Ther 2012;36:644649.

69. Lambert AA, Lam JO, Paik JJ, Ugarte-Gil C, Drummond MB, Crowell TA. Risk of community-acquired pneumonia with outpatient protonpump inhibitor therapy: a systematic review and meta-analysis. PLoS One 2015;10:e0128004.

70. Ito T, Jensen RT. Association of long-term proton pump inhibitor therapy with bone fractures and effects on absorption of calcium, vitamin B12, iron, and magnesium. Curr Gastroenterol Rep 2010;12:448-457.

71. Yearsley KA, Gilby LJ, Ramadas AV, Kubiak EM, Fone DL, Allison MC. Proton pump inhibitor therapy is a risk factor for Clostridium difficile-associated diarrhoea. Aliment Pharmacol Ther 2006;24:613-619.

72. Tai SY, Chien CY, Wu DC, et al. Risk of dementia from proton pump inhibitor use in Asian population: a nationwide cohort study in Taiwan. PLoS One 2017;12:e0171006.

73. Keeling DJ, Laing SM, Senn-Bilfinger J. SCH 28080 is a lumenally acting, $\mathrm{K}^{+}$-site inhibitor of the gastric $\left(\mathrm{H}^{+}+\mathrm{K}^{+}\right)$-ATPase. Biochem Pharmacol 1988;37:2231-2236.

74. Dent J, Kahrilas PJ, Hatlebakk J, et al. A randomized, comparative trial of a potassium-competitive acid blocker (AZD0865) and esomeprazole for the treatment of patients with nonerosive reflux disease. Am J Gastroenterol 2008;103:20-26.

75. Echizen H. The first-in-class potassium-competitive acid blocker, vonoprazan fumarate: pharmacokinetic and pharmacodynamic considerations. Clin Pharmacokinet 2016;55:409-418.

76. Kojima Y, Takeuchi T, Sanomura M, et al. Does the novel potassiumcompetitive acid blocker vonoprazan cause more hypergastrinemia than conventional proton pump inhibitors? a multicenter prospective crosssectional study. Digestion 2018;97:70-75.

77. Miyamoto S, Matsuno Y, Kato M, et al. Parietal cell protrusions and dilated oxyntic glands from use of vonoprazan. Am J Gastroenterol 2017;112:1899-1901.

78. Takimoto M, Tomita T, Yamasaki T, et al. Effect of vonoprazan, a potassium-competitive acid blocker, on the ${ }^{13} \mathrm{C}$-urea breath test in Helicobacter pylori-positive patients. Dig Dis Sci 2017;62:739-745. 